\title{
Gate Sizing in the Presence of Gate Switching Activity and Input Vector Control
}

\author{
Nathaniel A. Conos, Saro Meguerdichian, and Miodrag Potkonjak \\ Computer Science Department \\ University of California, Los Angeles \\ \{conos, saro, miodrag\}@cs.ucla.edu
}

\begin{abstract}
We introduce a novel gate sizing approach that considers both the gate switching activity (SA) and gate input vector control leakage (IVC). We first extract SA using simulation and find promising input vectors. Next, in an iterative framework, we interchangeably conduct gate sizing and refining the IVC. As dictated by the new objective function, our algorithm conducts iterative gate freezing and unlocking with cut-based search for the most beneficial gate sizes under delay constraints. We evaluate our approach on standard benchmarks in $45 \mathrm{~nm}$ technology, showing promising improvement, achieving up to $62 \%$ (29\% avg.) energy savings compared to the traditional objective function.
\end{abstract}

\section{INTRODUCTION}

Gate sizing is a powerful optimization technique used to minimize power and/or area under strict timing constraints by altering the widths of transistors in gates. Gate sizing has been extensively studied over the past three decades [1][2][4] and several approaches have been proposed. Previous approaches, however, do not consider switching activity (SA) and the impact of input vector control leakage (IVC), which greatly impact the overall optimization strategy. Furthermore, gate sizing is often combined with dual or multi-threshold techniques which further increases the importance of accurate power and delay modeling. As a result, the modern design flow imposes a number of modeling and optimization challenges

A major challenge is the simplification of timing and power models, which may lead to suboptimal solutions when mapping out to real designs. Accounting for accurate gate and interconnect delay and its dependencies on capacitive load slew are often ignored [4]. Additionally, nominal gate switching activity and/or average gate leakage are generally assumed in previous works limiting the potential improvements by accounting for realistic operating conditions. Moreover, previous approaches are either dynamic or leakage powercentric in their optimization flows, which do not address the varying application usage characteristics present in highperformance systems (e.g., data-centers, super-computing) to energy constrained mobile devices (e.g., tablets, smart-phones).

Cell library-based optimization has emerged as the de facto standard for modeling power and delay of a circuit design. Many previous approaches, however, utilize simplified timing models by assuming convex and/or linear delay and power models [3]. Empirical analysis has shown that accurate timing models are non-linear/non-convex. Furthermore, optimizing circuit designs using a discrete cell library, however, leads to solving an NP-Hard problem [5]. As a result, many heuristics have been developed in order to address the huge problem search space. A major drawback of these methods is that they require heavy parameter tuning and are difficult reproduce, since they are technology dependent, and are rooted to a set of sensitivity functions. These methods often perform iterative per-gate or per-group improvement are too compute intensive and are impractical to be applied on modern IC sizes, even with incremental updates. Furthermore, these approaches mainly perform local optimization (i.e., local-moves) and are susceptible to be trapped in local minimas [6].

The usage of modern cell libraries, however, have enabled the support various supply/threshold voltages, and drive strengths, enabling a rich performance and energy tradeoff to address the potentially vastly differing device usage characteristics. However, current tools do not account for realistic conditions into their objective functions (e.g., gate activity, duty cycle, input vector control), with respect to their applications, potentially impacting obtained results.

We improve state-of-the-art sizing methodologies by simultaneously considering gate switching activity $(S A)$ and gate input vector control $(I V C)$. One of our key contributions is that we demonstrate significant benefits of incorporating actual gate SA and gate IVC in the objective function over the equivalent approach that only uses nominal and average values for switching and leakage weights, and show how the obtained solution varies when accounting varying duty cycles.

The focal point of our approach is a scalable gate sizing algorithm that considers gate $S A$ and $I V C$ leakage. The steps are to: 1) extract the SA of gates based on simulation of real workloads; and 2) conduct IVC to obtain the input vector that induces the lowest total leakage energy across all gates, and 3) an iterative gate sizing approach freezes maximally-constrained gates (ones that are at high-power states as determined by SA and IVC) while searching for a sizing option that best improves the current picture. The objective function in step 3 to be considered at the iteration depends on the types of options available and their impacts on both delay and energy. The algorithm prevents the algorithm from reaching a local minima by freezing gates as they are sized until all gates have been frozen, then unfreezes all gates, reconducts IVC (since new gates may be energy-dominant), and reiterates steps 2 and 3 until the solution converges or the delay constraint cannot be met.

We evaluate our approach on benchmarks included in ISCAS-85/89, ITC99 and arithmetic units. Our results indicate over $62 \%$ (29\% avg.) energy improvement over a method that 


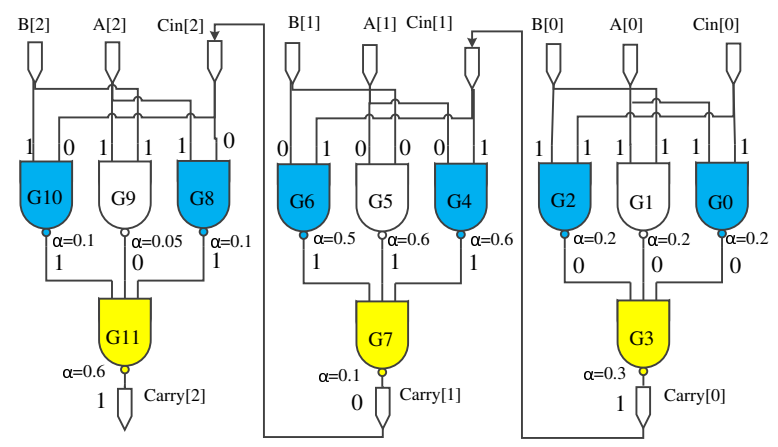

Fig. 1: Carry propagation for 3-bit carry-ripple adder.

assumes nominal $S A$ and $I V C$, demonstrating that gate $S A$ and $I V C$ play an major role in the guiding sizing decisions.

\section{Motivation}

We begin by providing a small realistic example demonstrating the importance of considering both SA and IVC in the gate sizing optimization process. Consider the carry propagation of a 3-bit carry-ripple adder, shown in Figure 1. Assume that 2- and 3-input NAND gates have input-dependent leakage power consumption values for two possible sizes, small (X1) and large (X2), shown in Table I. Also assume that the given input vectors $(A=101, B=101$, and $C_{i n}=1$ ) are realized throughout the entire duration of the application. Figure 1 shows the input vectors to each gate. Therefore, the overall leakage power of the circuit is 288 $\mathrm{nW}$. For simplicity of the example, ignoring load and slew dependencies, assume that all gates have delay of $10 \mathrm{ps}$ at size $\mathrm{X} 1$ and $5 \mathrm{ps}$ at size $\mathrm{X} 2$. Finally, assume that at the beginning of the optimization process, all gates are nominally sized to size $\mathrm{X} 1$. Therefore, there are eight nominal critical paths (colored), $\{G 0, G 2\} \rightarrow G 3 \rightarrow\{G 4, G 6\} \rightarrow G 7 \rightarrow\{G 8, G 10\} \rightarrow G 11$, with nominal delay 60 ps. Consequently, total leakage energy of the circuit is $1.73 \times 10^{-17} \mathrm{~J}$.

As an example, consider a delay constraint of $55 \mathrm{ps,} \mathrm{it} \mathrm{is}$ clear that one of gates G3, G7, or G11 should be sized up to $\mathrm{X} 2$, as all critical paths pass through these bottleneck gates and decrease the delay of each of these gates will decrease the overall delay. A traditional approach to gate sizing would consider these gates equally. In other words, increasing the size of either would decrease delay and increase switching and leakage power by the same amounts. However, from Table I, we see that the leakage power of a gate, due to transistor stacking, strongly depends on its applied inputs, with up to a 43X difference between the lowest-leakage state (input vector "100": $1.29 \mathrm{nW}$ ) and highest-leakage state (input vector "111": $55.8 \mathrm{nW}$ ) of a 3-input NAND gate. Furthermore, switching energy of a gate is directly proportional to its activity factor, or the likelihood that the gate will switch. Therefore, because the gates have both different applied input vectors and different activity factors, sizing up each one will have a different effect on overall power and energy consumption, so they should not be weighted equally in the optimization process.

First, consider the case where the duty cycle of the adder is low and therefore leakage energy dominates. We can determine from Table I that increasing the size of gates G3, G7, or
TABLE I: NAND gate leakage values $(n W)$ for two sizes $(\mathrm{X} 1, \mathrm{X} 2)$ based on input vector control (IVC) from a single threshold 45-nm cell library [7], where min and max leakage states are represented by bold and italicized fonts, respectively.

\begin{tabular}{|c|cc|}
\hline & \multicolumn{3}{|c|}{ NAND-3 } \\
\hline IVC & X1 & X2 \\
\hline \hline 000 & 3.32 & 13.28 \\
\hline 001 & 18.18 & 72.73 \\
\hline 010 & 4.21 & 16.84 \\
\hline 011 & 39.49 & 157.97 \\
\hline $\mathbf{1 0 0}$ & $\mathbf{1 . 2 9}$ & $\mathbf{5 . 1 5}$ \\
\hline 101 & 18.78 & 75.13 \\
\hline 110 & 3.76 & 15.04 \\
\hline 111 & 55.8 & 223.22 \\
\hline
\end{tabular}

\begin{tabular}{|c|cc|}
\hline & NAND-2 & \\
\hline IVC & X1 & X2 \\
\hline \hline $\mathbf{0 0}$ & $\mathbf{3 . 4 8}$ & $\mathbf{1 3 . 9 3}$ \\
\hline 01 & 24.8 & 99.2 \\
\hline 10 & 4.09 & 16.34 \\
\hline 11 & 37.21 & 148.83 \\
\hline
\end{tabular}

G11 will increase leakage power by $9.96 \mathrm{nW}, 167.42 \mathrm{nW}$, or $56.35 \mathrm{nW}$, respectively, while decreasing the overall delay by 5 ps. Therefore, the optimal decision is to increase the size of gate G3, which will have minimal impact on leakage energy, increasing leakage power to $298 \mathrm{nW}$ and decreasing leakage energy to $1.64 \times 10^{-17} \mathrm{~J}$. Increasing the size of G7 would instead increase leakage power to $455 \mathrm{nW}$, increasing leakage energy to $2.50 \times 10^{-17} \mathrm{~J}$. Thus, considering IVC in this example in the optimization algorithm can improve the energy by roughly $60 \%$.

Now, consider the high duty cycle scenario, where switching energy is the dominant factor. Again, for simplicity, assume that all gates consume $10 \mathrm{~nJ}$ and $20 \mathrm{~nJ}$ of switching energy at nominal activity factor 1.0 for a given application at sizes $\mathrm{X} 1$ and X2, respectively. Figure 1 shows the activity factors $(\alpha)$ for each gate. Therefore, overall switching energy consumption at the nominal size is $35.5 \mathrm{~nJ}$. In this case, increasing the size of gate G7 is the optimal decision, since it has the lowest activity factor and consumes less switching energy than when up-sizing either G3 or G11. In fact, this decision results in a switching energy of $36.5 \mathrm{~nJ}$, whereas increasing the size of G11 would result in a switching energy of $41.5 \mathrm{~nJ}$. Therefore, the decision that considers SA performs roughly $14 \%$ better.

To present these motivations, we have made a number of assumptions that when relaxed make the optimization much more complex in practice. It is reasonable to assume that additional information (gate switching, input vector state) can be readily obtained by modern CAD tools and/or by implementing a simple gate-level simulator. Such information is beneficial since it enables the simultaneous consideration of low duty cycle and high duty cycle scenarios, as in real use cases at current and pending deep-submicron feature sizes, leakage and switching may both have significant impacts on overall energy. For example, sizing up G7 in the high duty cycle scenario may in reality not be optimal, since its input gates have higher values for $\alpha$ than, the input gates of G3, and thus their switching energies would increase by larger factors. Thus, this IVC depends on how the circuit is sized and its dutycycle. Therefore, a feedback loop exists between gate sizing and IVC that must be addressed simultaneously during the optimization. The simple example here demonstrates that both IVC and SA are crucial considerations in gate sizing for energy optimization in the presence of delay deadlines. 


\section{RELATED WORK}

We now cover a set of related gate sizing approaches that have considered a variant of $S A$ or $I V C$. Several approaches exist that address continuous and discrete gate sizing. Common methods to solve the gate sizing problem have been convex optimization [3], Lagrangian Relaxation [1][2][18], and gradient and sensitivity-based optimization [8][19].

Gate sizing methods have also been combined with $V_{d d}$ and $V_{t h}$ assignment to minimize power under various gate $S A$ ratios [9][10]. These works, however, have only considered average leakage values when accounting for leakage and have not explored real application activity factors when considering gate switching activity. Leakage minimization using $I V C$ is a popular technique for due to its strong dependency on the input vector state [11]. IVC and gate replacement techniques have also been combined [12] by replacing gates at their worse-case leakage state with equivalent gates with lower leakage power. The technique is further combined with circuit aging in preand-post silicon phases. [13][20]. IVC has also been explored in the presence of uncertainty [14].

To the best of our knowledge, we are the first to consider gate sizing in the presence of both $S A, I V C$, and duty cycle. Prior approaches have at most considered one or two terms accurately [17], and/or do not differentiate between the duty cycle with respect to switching and leakage energy weights, leaving many approaches to be either dynamic or leakage power-centric. For example, the state-of-the-art gate sizing contest considers only nominal leakage power [4]. Our technique minimizes total energy, such that both the switching and leakage energy components are accurately accounted for in accordance to their usage or duty cycle.

\section{Cell Library Energy and Delay}

The total energy of a CMOS integrated circuit can be characterized into two main components: 1) dynamic (switching) energy due to charging of input pin/output load capacitance's; and 2) static (leakage) energy, which we model from the dominant sub-threshold leakage and gate leakage currents. Thus, the total energy consumed can be computed as:

$$
\begin{aligned}
E_{\text {total }} & =E_{\text {switch }}+E_{\text {leak }} \\
E_{\text {switch }} & =\sum_{i}^{N} e s\left(g_{i}\right), \quad E_{\text {leak }}=\sum_{i}^{N} \operatorname{el}\left(g_{i}\right)
\end{aligned}
$$

$e s$ and $e l$ represent the switching and leakage energies, respectively, for gate $g_{i}$. es is the product between probability that a gate's input pin $j$ will switch, $\alpha$ (SA), and the estimated fullcycle $\left(e_{f c}\right)$ power consumed from propagating a signal from input pin $j$ to output pin $k$. el is the sum of leakage energies consumed at each possible leakage state of a gate, which is also dependent the ratio of the total time spent at each leakage state for both active and standby (idle) periods. The total time $(T)$ is directly proportional to product of the circuit delay $(D)$ and total cycles, where $D$ represents the critical output-pin arrival time (rise or fall) of a primary output gate $o t^{r, f}\left(g_{i}\right)$ :

$$
D=\max \left(\text { ot }^{r, f}\left(g_{i}\right)\right) \quad \text { s.t. } g_{i} \in G_{\text {out }}
$$

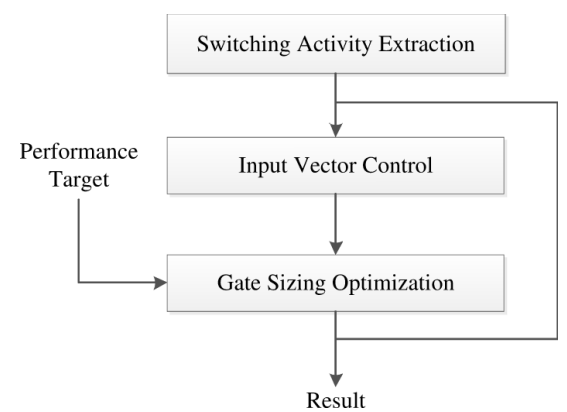

Fig. 2: Gate sizing optimization flow.

$G_{\text {out }}$ represents a circuit's set of primary output gates. Therefore, the delay of a circuit can be determined by solving:

$$
\begin{array}{r}
o t^{r, f}\left(g_{i}\right)=d l^{r, f}\left(g_{i}\right)+\max \left(\text { ot }\left(\operatorname{fin}_{j}^{f, r}\right)\right) \\
\text { s.t. fin } \in F I_{i}
\end{array}
$$

$\operatorname{fin}_{j}^{f, r}$ is the fall, rise arrival time of a fan-in gate $j$ in the set $F I_{i}$ of gate $g_{i}$. Note that the propagation of delay depends on the unateness assumption. For simplicity, we assume all cells are negative unate, thus, rise $(r)$ and fall $(f)$ gate delays are propagated as assumed to the next stage.

We use a cell table library look-up as [4] to model gate rise and fall delay $\left(d l^{r, f}\right)$ as a function of its input slew (transition time), and driving load. However, we use an alternate 45$\mathrm{nm}$ cell library (Nandgate) [7] to account for switching and input vector dependent leakage power, which are obtained in a similar look-up table fashion, provided per-input pin accurate switching, and input vector state probabilities, which can be obtained using gate-level simulation.

\section{Technical Approach}

Our gate sizing procedure is composed of three major phases (Figure 2). The first phase extracts gate switching activity factors $(S A)$ for a given circuit by performing eventdriven gate simulation from a set of input bit vectors. Figure 3 illustrates an example $S A$ extraction for a carry-look-ahead unit (cla4) from two applications (mpeg2enc/dec). The second step identifies a primary input bit vector that places gates in low leakage states in order to minimize the total energy of the circuit, which accounts for leakage consumption for both active (obtained from SA) and idle periods. IVC techniques range from random simulation to satisfiability (SAT) and model counting-based formulations. The final component is the gate sizing algorithm, where the goal is to minimize total energy consumption under a delay constraint. The approach is iterative; at each iteration, gates are either frozen or unlocked based on their leakage (IVC) and switching (SA) impact, while a search is conducted for the most beneficial current move.

Our algorithm is sensitivity-based in nature in terms of determining which move or set of moves to perform. A gate sizing move can have 1 of 3 effects (increase, decrease, have no effect) on 2 parameters (energy and delay), leading to a total of 9 separate possible classes for a move. The algorithm classifies each move to a class and enforces a priority in terms of selecting a move that has higher precedence. There are three precedence levels, where level 1 is the highest priority. Moves 


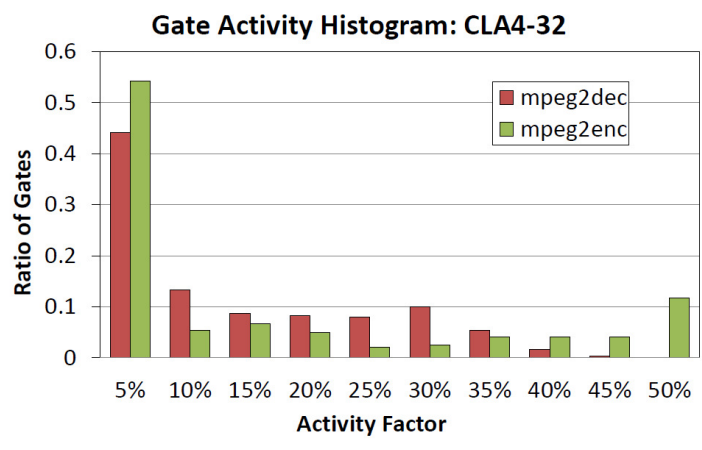

Fig. 3: Gate switching activity for a 32-bit CLA circuit when using real mpeg2enc/dec application input stimulus. Shown are varying distribution of gate activity within a circuit and across two applications.

that improve both parameters are at precedence 1 , moves that improve just one parameter and do not affect the other are at precedence 2, and moves that improve one parameter at the expense of degrading another are of precedence 3 . Note that moves that degrade both parameters are never selected. Each precedence level has its own objective function for selecting the best move: 1) the product of the respective improvements; 2) the single improvement; and 3 ) the normalized ratio of improvement and degradation.

The algorithm considers a cut of $M$ gates at a time and restricts one gate to be sized per group visit. Once a size move is committed, the gate is locked and is no longer considered within that phase. The completion of a phase is defined as having locked all gates, or having no more acceptable moves among sizable gates that improve the objective function. The algorithm terminates after the solution converges or if a target delay $\left(D_{\text {target }}\right)$ can not be met after a number of phases. All gates are unlocked before the start of each new sizing phase.

The algorithm initially freezes the top $K$ energy-critical gates by setting them to their minimal sizes at the beginning of the phase. We note that this initial set potentially restricts some delay critical gates, as improving the delay of these gates may be required in meeting a deadline. To relax this constraint (i.e., if a solution cannot be obtained), $K$ is relaxed through a locking threshold ratio $\gamma$, where a new $K$ is computed (e.g., $K=K^{\prime} \cdot \gamma$ ), thereby, enabling potentially more delay critical gates to be reduced. It is crucial to identify the top $K$ energy-critical gate, which in turn depends on both SA and IVC; this maximally-constrained gate locking is one of the key innovations of the approach, and prevents being trapped at a local minima by encouraging global circuit optimization.

We utilize an epsilon tree to minimize circuit delay updates $\left(\epsilon_{\text {path }}\right)$, which consists of gates that were on the critical path during the last accurate delay computation (Figure 4a). Since the critical path may change during optimization, we also include the immediate fan-out gates of each critical gate (e.g., nodes 1 and 3), fan-in nodes may be added for greater accuracy as their slews may also impact timing propagation. The figure shows bold-outlined nodes (e.g., 7, 8, 9, 5 and 6) are the primary outputs $\left(G_{\text {out }}\right)$, and are transitively connected to at least one node belonging to the critical path (e.g., 0, 2, 4, $8)$. Thus, the delay cost of sizing a gate on the $\epsilon_{\text {path }}$ can be estimated by the sum of its $\delta_{i}$ respect to the target delay

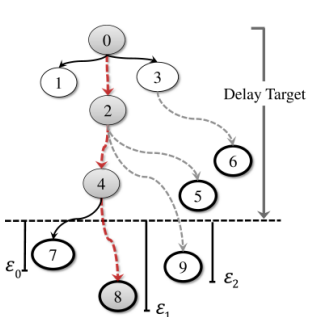

(a)

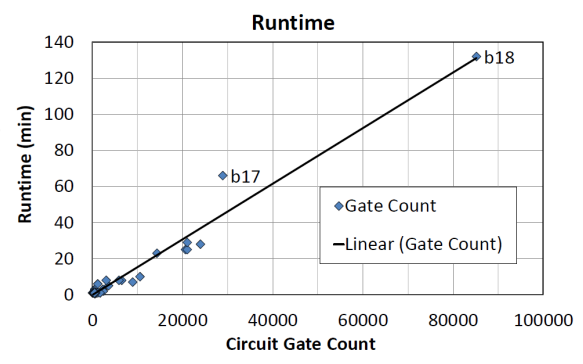

(b)
Fig. 4: (a) An example of $\epsilon$ critical path $\left(\epsilon_{\text {path }}\right)$; the critical path in red; transitive fan-out output nodes in bold outlines; and $\epsilon_{i}$ corresponds to the absolute delay difference w.r.t to the target delay used for estimating delay cost of a move. (b) The linear run-time of the new gate sizing approach.

$\left(D_{\text {target }}\right)$ is used to estimate the delay impact of each move via a delay cost formula, as shown below:

$$
D_{\text {cost }}=\sum_{i}^{\left|G_{\text {out }}\right|}\left(\delta_{i}-D_{\text {target }}\right)^{2}
$$

This formulation enables very efficient delay estimation by only considering the delay impact of a small subset of gates at a time. A drawback of this approach, however, is that a potentially new critical path may emerge. This remains to be a major challenge for existing gate sizing techniques that attempt to maintain delay accuracy during optimization [8][18][19]. To address this issue, the frequency of delay updates can be increased by adjusting $M$ and $\gamma$ to be larger values, as we have done. These parameters can be adjusted, to trade-off accuracy vs run-time. Our used values of $M$ and $\gamma$ achieved a delay accuracy to be within 5\%, while achieving linear run-time scaling with respect to circuit size (Figure 4b).

\section{EXPERIMENTAL RESULTS}

We evaluated our gate sizing approach on a set of benchmarks in ISCAS-85/89, ITC-99 suites, as well as integer arithmetic units consisting of adders (carry ripple, carry-lookahead, Kogge-Stone) and multipliers (array, Dadda). All units were synthesized using a single threshold (HVt) 45-nm open cell library from [7] under the typical cell configuration. An in-house timing/power engine was implemented in $\mathrm{C}++$ and was correlated to an industrial tool, Synopsys PrimeTime, to be within $10^{-3} \mathrm{ps}$. All results were optimized using identical rules such as ensuring no slew or load violations exists in the final design, as presented in [4]. The only differences in our framework is the choice in cell library, which was done in order to enable accurate IVC computations, as well as the choice of circuit benchmarks. In handling slew and load violations, we adopt an iterative approach as proposed in [19].

The SA of gates and IVC for each circuit were obtained from simulation of random input vectors. However, real application switching activity factors were obtained from mpeg2enc/dec benchmarks from recorded operand values from each unit type, running ARM7TDMI-ISA mpeg2-enc/dec traces [15][16]. The initial simulation parameters set were, $K$ $=25 \%, M=$ twice the length of average critical path, $\gamma=$ 0.2 , and were fixed across all benchmarks. The delay target for each circuit was set as the median between the achieved delay when all gates were set to their maximal size, and the 


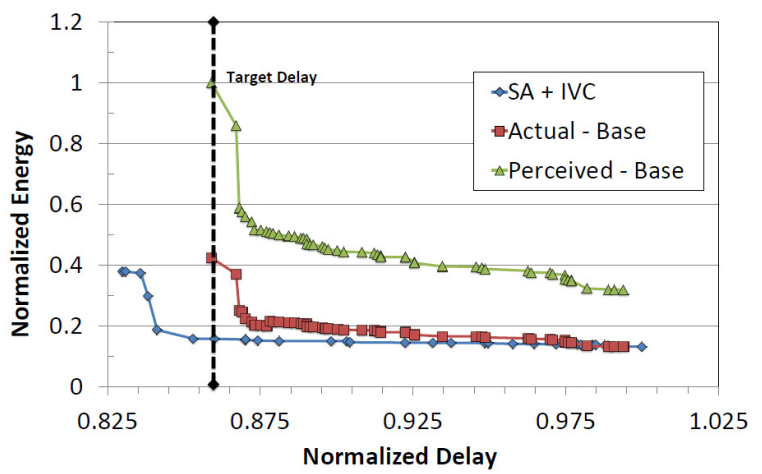

Fig. 5: Energy vs delay plot of c2670. The $S A+I V C$ approach consistently outperforms the Base method.

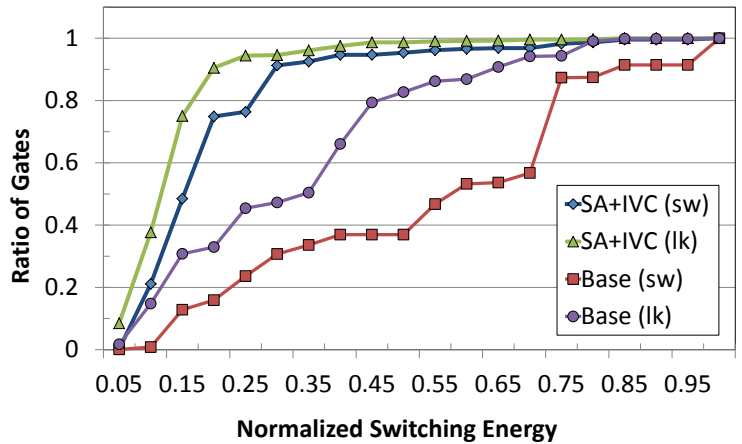

Fig. 6: Cumulative distribution of leakage and switching energy after sizing for gates in c2670. The accurate $S A+I V C$ approach results in a higher percentage of gates at lower energy.

achieved delay when all gates are at their minimal size. Five duty cycle scenarios $(\mathrm{D} 0=10 \%, \mathrm{D} 1=20 \%, \mathrm{D} 2=33 \%, \mathrm{D} 3=50 \%$, and $\mathrm{D} 4=100 \%)$ were considered.

We evaluate our sizing algorithm under two gate sizing assumptions: 1) SA+IVC, which considers gate switching activity factors and input vector control in the objective function; and 2) Base, where the objective function uses only nominal gate switching (50\%) and average gate leakage values for total energy computation. Table II compares the two methods, where Max (\%) savings corresponds to the maximum energy improvement achieved over the Base method across the five duty cycle cases (D0 to D4) for each circuit under the same timing constraint. As expected, the maximum improvement observed varies across duty cycles and circuits, motivating the advantage of utilizing accurate power and delay knowledge.

Table IV provides overall energy improvements across the benchmarks suite. The results generated by the new approach achieved a max energy improvement of $62 \%$ for circuit c 2670 and $29 \%$ average overall for the same delay. Figure 5 provides a normalized energy and delay plot for c2670, which illustrates the advantage of using more accurate power and delay information. A delay of 0.87 shows that the Perceived (green) energy deviates from the trend of the Actual (red) energy plot. In performing move-trace analysis, we noted that Base method caused the algorithm to over-size a few selected critical paths and encountered a timing wall much earlier, where as $S A+I V C$ was able to efficiently trade-off delay for an additional 0.05 delay units, as shown.
TABLE IV: Overall energy savings with respect to benchmark suite.

\begin{tabular}{|c|c|c|c|c|}
\hline $\begin{array}{c}\text { Benchmark } \\
\text { Suite }\end{array}$ & $\begin{array}{c}\text { Max Tot } \\
(\%)\end{array}$ & $\begin{array}{c}\text { Avg. Tot } \\
(\%)\end{array}$ & $\begin{array}{c}\text { Avg. Sw } \\
(\%)\end{array}$ & $\begin{array}{c}\text { Avg. Lk } \\
(\%)\end{array}$ \\
\hline ISCAS-85 & 62.64 & 29.70 & 30.58 & 26.74 \\
ISCAS-89 & 53.20 & 28.33 & 29.99 & 26.96 \\
ITC-99 & 58.83 & 29.23 & 30.90 & 24.15 \\
Arith & 57.19 & 30.48 & 33.23 & 33.15 \\
\hline
\end{tabular}

Figure 6 shows a cumulative distribution of gate switching and leakage energies of the max improved result for $S A+$ $I V C$ over Base for circuit c2670. Our approach shows that accurate knowledge enabled the algorithm to efficiently guide the circuit to a lower energy state, as shown with higher percentage of gates falling under lower energy profiles for both leakage and switching energy. This is important to note since due to the difficulty of comparing gate sizing algorithms, many existing algorithms are sensitivity-based in nature, thus, the ability to guide an algorithm to determine more promising "moves" greatly impacts the optimization procedure.

Table III presents results comparing the minimal configuration found by $S A+I V C$ and the perceived minimal configuration obtained by Base. The minimum energy configuration determined was cla432 and dad8, optimized under the same timing constraints determined by the the multiplier. For these configurations, our approach shows additional savings in both leakage and switching categories where the majority of the savings for both cases (15\% mpeg2enc, $25 \%$ mpeg2dec) were achieved by the multiplier circuit.

\section{CONCLUSION}

We present a new gate sizing approach that includes the switching activity (SA) and input vector control (IVC) to minimize overall energy. The new objective function has several ramifications on the optimization procedure, including the need for reiteration between gate sizing and input vector selection and freezing and unlocking of high-power gates. On a comprehensive set of benchmarks, from ISCAS-85/89, ITC99, and arithmetic units, synthesized using $45 \mathrm{~nm}$ technology, we reduce average actual energy consumption by $30 \%$. The approach is generic in the sense that thermal impacts and multi- $V_{t h}$ can be easily addressed using the new optimization procedure.

\section{REFERENCES}

[1] H. Shiyan et al., "Gate Sizing For Cell Library-Based Designs," DAC pp. 847-852, 2007.

[2] M. M. Ozdal et al., "Gate sizing and device technology selection algorithms for high-performance industrial designs," ICCAD, pp. 724731, 2011.

[3] S. Joshi, "An Efficient Method for Large-Scale Gate Sizing," TCSI, pp. 2760-2773, 2008.

[4] M. M. Ozdal et al., "The ISPD -2012 Discrete Cell Sizing Contest and Benchmark Suite," In Proceedings of ISPD pp. 161-164, 2012

[5] W. N. Li, "Strongly NP-hard discrete gate-sizing problems," ICCD, pp.1045-1051, 1993.

[6] A. Agarwal et al., "Statistical timing based optimization using gate sizing," DAC, pp. 400-405, 2005.

[7] Nangate FreePDK45-nm Library, http://www.si2.org/, 2011.

[8] O. Coudert, "Gate sizing for constrained delay/power/area optimization," VLSI, pp. 465-472, 1997. 
TABLE II: Energy improvements when considering gate $S A$ and $I V C$ during the gate sizing procedure over the Base method. The obtained switching and leakage energies are presented for the $S A+I V C$. The maximum energy deltas $(\Delta \%)$, corresponds to the max difference in energy profile "perceived" by the Base method during optimization.

\begin{tabular}{|c|c|c|c|c|c|c|c|c|c|c|}
\hline & & \multicolumn{3}{|c|}{ Max Energy Improvement } & \multicolumn{4}{|c|}{$S A+I V C$} & & \\
\hline Circuit & No. Gates & $\begin{array}{c}\text { Total } \\
(\%)\end{array}$ & $\begin{array}{l}\text { Sw } \\
(\%)\end{array}$ & $\begin{array}{c}\mathrm{Lk} \\
(\%)\end{array}$ & $\begin{array}{l}\text { Sw } \\
(\mu \mathrm{J})\end{array}$ & $\begin{array}{l}\text { Max. } \\
(\Delta \%)\end{array}$ & $\begin{array}{c}\mathrm{Lk} \\
(\mu \mathrm{J})\end{array}$ & $\begin{array}{l}\text { Max. } \\
(\Delta \%)\end{array}$ & $\begin{array}{l}\text { Duty } \\
\text { Cycle }\end{array}$ & $\begin{array}{c}\text { Delay } \\
\text { (ns) }\end{array}$ \\
\hline $\mathrm{c} 880$ & 383 & 8.14 & 8.16 & 8.05 & 125.11 & 31.22 & 15.75 & 3.01 & D2 & 0.73 \\
\hline c1355 & 554 & 14.35 & 15.01 & 13.26 & 264.13 & 31.12 & 164.79 & 15.19 & D1 & 0.74 \\
\hline c1908 & 932 & 13.41 & 13.72 & 9.41 & 405.7 & 29.57 & 32.06 & 6.75 & D4 & 1.14 \\
\hline c7552 & 3568 & 43.65 & 44.02 & 41.32 & 1685 & 30.27 & 284.6 & 3.21 & D2 & 1.20 \\
\hline c5315 & 2330 & 58.72 & 59.1 & 54.57 & 855.6 & 32.93 & 87.58 & 3.19 & D4 & 1.34 \\
\hline c432 & 168 & 30.15 & 35.24 & 22.35 & 68.54 & 33.15 & 53.55 & 23.67 & D1 & 0.62 \\
\hline c2670 & 1202 & 62.64 & 62.74 & 60.78 & 407.6 & 31.91 & 24.62 & 1.39 & D4 & 0.85 \\
\hline c3540 & 1703 & 28.84 & 29.66 & 24.14 & 799.1 & 31.09 & 152.2 & 2.47 & D2 & 0.79 \\
\hline s1488 & 698 & 46.38 & 46.49 & 44.81 & 182.6 & 35.54 & 14.09 & 12.05 & $\overline{\mathrm{D} 2}$ & 0.42 \\
\hline s1494 & 692 & 35.5 & 36.08 & 33.84 & 283.9 & 36.36 & 103.3 & 12.55 & D1 & 0.42 \\
\hline s15850 & 10547 & 31.34 & 30.76 & 34.48 & 3803 & 33.69 & 662.1 & 4.98 & D3 & 2.22 \\
\hline s838 & 473 & 32.49 & 38.89 & 27.17 & 139.3 & 31.76 & 199.8 & 10.84 & D1 & 1.64 \\
\hline s5378 & 3054 & 16.76 & 30.08 & 15.06 & 826.6 & 36.2 & 7876 & 26.34 & D0 & 0.68 \\
\hline s9234 & 5897 & 50.19 & 50.52 & 47.23 & 2041 & 32.51 & 242.5 & 4.43 & D3 & 1.50 \\
\hline s38417 & 23963 & 53.2 & 53.85 & 46.17 & 6661 & 30.65 & 714.2 & 8.58 & D3 & 1.29 \\
\hline s35932 & 21035 & 22.27 & 22.31 & 21.25 & 8317 & 29.28 & 332.1 & 37.26 & D3 & 0.80 \\
\hline s38584 & 18161 & 29.01 & 31.52 & 28.75 & 5820 & 32.24 & 59720 & 4.77 & D0 & 1.52 \\
\hline$\overline{\mathrm{b} 10}$ & $\overline{204}$ & $\bar{~} 44.69$ & 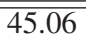 & 32.43 & 40.56 & 34.45 & 1.55 & 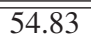 & $\overline{\mathrm{D} 2}$ & 0.36 \\
\hline b11 & 633 & 35.53 & 35.79 & 31.74 & 250.9 & 32.37 & 18.77 & 61.52 & D2 & 1.10 \\
\hline b12 & 1183 & 22.91 & 27.89 & 3.31 & 314.0 & 37.03 & 107.3 & 60.54 & D1 & 0.61 \\
\hline b13 & 375 & 22.05 & 22.78 & 15.01 & 161.23 & 31.85 & 18.4 & 55.33 & D1 & 0.33 \\
\hline b14 & 6498 & 28.42 & 28.65 & 26.21 & 3024 & 33.22 & 320.7 & 54.94 & D2 & 1.31 \\
\hline b15 & 8920 & 27.62 & 27.7 & 26.75 & 1666 & 38.99 & 166.1 & 54.24 & D3 & 1.58 \\
\hline b17 & 28911 & 21.25 & 25.29 & 20.79 & 8.53 & 38.76 & 80.58 & 55.99 & D3 & 1.68 \\
\hline b18 & 85188 & 7.02 & 8.67 & 5.96 & 44.54 & 37.84 & 71.4 & 54.72 & D1 & 2.10 \\
\hline b20 & 14322 & 27.85 & 28.57 & 24.07 & 3.66 & 33.2 & 0.74 & 55.8 & D2 & 2.08 \\
\hline cra32 & 225 & 38.32 & 45.14 & 37.76 & 6.72 & 44.90 & 92.22 & 32.09 & D0 & 2.08 \\
\hline cla432 & 305 & 22.25 & 25.02 & 12.18 & 10.18 & 42.87 & 3.28 & 13.62 & D2 & 0.35 \\
\hline ks32 & 611 & 24.2 & 23.18 & 24.63 & 17.64 & 44.76 & 40.83 & 14.6 & D0 & 0.30 \\
\hline arr8 & 512 & 35.96 & 36.3 & 23.18 & 178.5 & 34.69 & 22.84 & 21.29 & D1 & 0.81 \\
\hline dad8 & 542 & 30.35 & 30.99 & 36.33 & 101.3 & 35.83 & 9.12 & 11.98 & D2 & 0.62 \\
\hline
\end{tabular}

TABLE III: Energy improvement of $(S A+I V C)$ over Base using extracted gate switching activity and input vector control from mpeg2enc/dec applications assuming a (D2) "33\%" duty cycle. The units represent an single-adder (32b) and multiplier (8b) configuration of an ARM7TDMI core [16].

\begin{tabular}{|c|ccc|cccc|cccc|}
\hline & \multicolumn{6}{|c|}{ Energy Improvement } & \multicolumn{4}{c|}{ cla432 } & \multicolumn{4}{c|}{ dad8 } \\
\hline & Total & Sw & Lk & Sw & Sw Imp. & Lk & Lk Imp. & Sw & Sw Imp. & Lk & Lk Imp. \\
Application & $(\%)$ & $(\%)$ & $(\%)$ & $(\mu \mathrm{J})$ & $(\%)$ & $(\mu \mathrm{J})$ & $(\%)$ & $(\mu \mathrm{J})$ & $(\%)$ & $(\mu \mathrm{J})$ & $(\%)$ \\
\hline mpeg2enc & 15.31 & 17.15 & 8.55 & 742.7 & 4.73 & 628.7 & 1.09 & 2267 & 20.61 & 498.6 & 13.92 \\
mpeg2dec & 25.10 & 29.89 & 6.21 & 11.02 & 6.42 & 24.30 & 1.20 & 101.4 & 30.99 & 9.24 & 21.58 \\
\hline
\end{tabular}

[9] A. Srivastava et al., "Power minimization using simultaneous gate sizing, dual-Vdd and dual-Vth assignment," DAC, pp. 783-787, 2004.

[10] H. Yu-Hui et al., "Switching-activity driven gate sizing and Vth assignment for low power design," ASPDAC, pp. 24-27, 2006.

[11] A. Abdollahi et al., "Leakage current reduction in CMOS VLSI circuits by input vector control," VLSI, pp. 140-154, 2004.

[12] L. Yuan et al., "A combined gate replacement and input vector control approach for leakage current reduction," VLSI, pp. 173-182, 2006.

[13] Y. Wang et al., "Leakage power and circuit aging cooptimization by gate replacement techniques," VLSI, pp. 615-628, 2011.

[14] Y. Alkabani et al., "Input vector control for post-silicon leakage current minimization in the presence of manufacturing variability," DAC, pp. 606-609, 2008.

[15] C. Lee et al., "MediaBench: a tool for evaluating and synthesizing multimedia and communicatons systems," MICRO, pp. 330-335, 1997.

[16] T. Mudge et al., "The SimpleScalar-Arm power modeling project," http://eecs.umich.edu/panalyzer/.

[17] C. Tsui et al., "Minimizing the dynamic and subthreshold leakage power consumption using least leakage vector assisted technology mapping," VLSI Journal, pp. 76-86, 2008

[18] L. Li et al., "An efficient algorithm for library-based cell-type selection in high-performance low-power designs," ICCAD, pp. 226-232, 2012

[19] J. Hu et al., "Sensitivity-guided metaheuristics for accurate discrete gate sizing," ICCAD, pp. 233-239, 2012

[20] S. Wei et al., "Aging-based Leakage Energy Reduction in FPGAs," FPL, pp. $277-277,2013$ 